\title{
Coulometry and Signal Enhancement for Microfluidic Systems
}

\author{
Hiroaki Suzuki \\ University of Tsukuba, 1-1-1 Tennodai, Tsukuba, Ibaraki, 305-8573, Japan \\ hsuzuki@ims.tsukuba.ac.jp
}

\begin{abstract}
:
In analyzing components in a solution of a very small volume, coulometry is advantageous. Sensitivity and detection limit can be improved by strategies including appropriate designing of the working electrode, coupling of the oxidation of an analyte with deposition of silver in a different flow channel, and replacement of a liquid junction that connects two flow channels with a metal wire. These techniques were actually effective in improving the performance of our microfabricated coulometric devices.
\end{abstract}

Key words: coulometry, hydrogen peroxide, silver, mixed potential, metal wire connection

\section{Introduction}

Electrochemical sensors facilitate miniaturization, batch-fabrication, and integration on a single chip. In many of previous microfabricated electrochemical devices, detection has been based on amperometry. However, achieving highly sensitive reliable detection becomes difficult with decreasing sample volume, because of the rapid depletion of the analyte. In this respect, coulometry is more advantageous. In coulometry, a generated charge, or current integrated over time, increases gradually, facilitating the measurement. Furthermore, unlike other techniques, calibration is not necessary if all the analytes are consumed exhaustively during the measurement. This is beneficial for batchfabricated disposable devices. In this presentation, recent progress of our techniques will be introduced.

\section{Coulometry in a microfluidic device}

Coulometry can be used for determining the analyte in a liquid plug confined in a flow channel [1]. The plug can be prepared using a T-junction and the volume can be measured precisely using changes in surface tension in a rhombus structure consisting of glass and poly (dimethylsiloxane). A thin-film three-electrode system was formed in a flow channel. We used different geometries for the working electrodes consisting an array of microelectrodes. Hydrogen peroxide was detected as a model analyte. A constricted flow channel structure was effective at efficiently consuming the analyte. Response profile changed depending on the geometry of the working electrode. With an increase in the generated charge while maintaining the background at a low level, the detection limit was lowered to $410 \mathrm{nM}$ using a working electrode structure.

\section{Coupling coulometry with metallization}

In the device, the generated charge increased monotonically as time elapsed and gradually leveled off as the analyte was depleted. However, background charge also increased as time elapsed. If the faradaic current is saturated within a very short time and the background charge can be minimized, both the sensitivity and detection limit should be improved significantly. To realize this, we used two flow channels $A$ and $B$ to convert the analyte into silver (Fig. 1A) [2]. The analyte was oxidized on
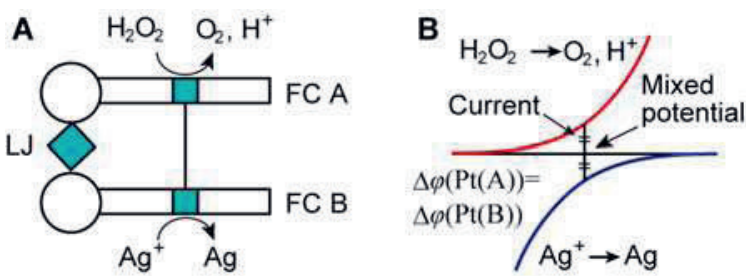

Fig. 1. Coulometry coupled with silver metallization. (A) Basic structure of the device and reactions that occur on two platinum electrodes in two flow channels. (B) Polarization curves of the related reactions and the mixed potential. The $x$ and $y$ axes indicate potential and current. LJ: liquid junction. FC: flow channel. $\Delta \varphi(\operatorname{Pt}(A))$ and $\Delta \varphi(\operatorname{Pt}(B))$ are the potentials of the platinum electrodes in flow channels $A$ and $B$. 
a part of a platinum electrode in flow channel $A$ and silver was deposited on the other part of the platinum electrode in flow channel $B$ at a mixed potential (Fig. 1B). We used different geometries for the electrodes in the two flow channels. Coulometric determination of the deposited silver showed a rapid increase in the generated charge. The measurement shortly after the start of the coulometry suppressed the background charge, resulting in significant lowering of the detection limit. The lower detection limit for $\mathrm{H}_{2} \mathrm{O}_{2}$ was 30 nM.

In the device, we used 26 disc microelectrodes to deposit silver. Although this may be necessary when the concentration of the analyte is high, increasing the number of electrodes only increases the background. To examine this effect, the change in the background was examined using $26,12,6$, or 2 microelectrodes. The background charge decreased with the decrease in the number of microelectrodes. On the other hand, the difference in current was small in the existence of $\mathrm{H}_{2} \mathrm{O}_{2}$, particulary with $\mathrm{H}_{2} \mathrm{O}_{2}$ of lower concentrations. The size of the microelectrodes also has an effect. To check this, 26 microelectrodes of different sizes were used. The background charge was reduced by decreasing the size of the electrodes. On the other hand, the charge was almost the same with $\mathrm{H}_{2} \mathrm{O}_{2}$. The result can be explained by efficient diffusion to the microelectrodes. The number and size of the microelectrodes can be chosen appropriately to improve the detection limit.

\section{Metal wire connection between flow channels for signal enhancement}

When a liquid junction is used to connect the two flow channels, the anodic and cathodic reactions are balanced and the electrode potential settles at the mixed potential. We then replaced the liquid junction with a silver wire with $\mathrm{AgCl}$ at both ends (Fig. 2A). For simplicity, we first assume that Ohmic drop in the flow channels and liquid-junction potential can be neglected. The relation between the potentials is given as follows.

$$
\begin{aligned}
& \Delta \varphi(\mathrm{Pt}(\mathrm{A}))-\Delta \varphi(\mathrm{Pt}(\mathrm{B}))= \\
& \Delta \varphi(\mathrm{MW}(\mathrm{A}))-\Delta \varphi(\mathrm{MW}(\mathrm{B}))=\Delta E
\end{aligned}
$$

Here, $\Delta \varphi(\mathrm{Pt}(\mathrm{A}))$ and $\Delta \varphi(\mathrm{Pt}(\mathrm{B}))$ are the potentials of the platinum electrodes in flow channels $A$ and $B$ with respect to the solutions, respectively. $\Delta \varphi(\mathrm{MW}(\mathrm{A}))$ and $\Delta \varphi(\mathrm{MW}(\mathrm{B}))$ are potentials of the two ends of the wire with respect to the solutions, respectively.

Fig. $2 B$ shows the relation in the case of $\Delta E>0$. Compared with the case of Fig. 1, it is
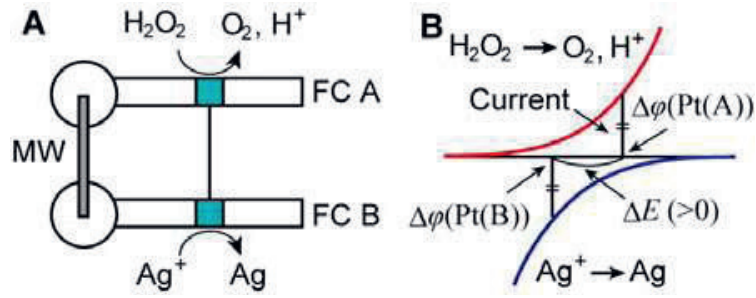

Fig. 2. Manipulating the electrode potentials by metal wire connection. (A) Coulometric device based on silver metallization with two flow channels connected with a metal wire. (B) Polarization curves of the reactions and related potentials. The $x$ and $y$ axes indicate potential and current. MW: metal wire. FC: flow channel.

anticipated that the current increases. To examine this effect, the potentials of the $\mathrm{Ag} / \mathrm{AgCl}$ electrodes were changed by changing the concentration of $\mathrm{Cl}^{-}$in the solutions. With two microelectrodes of $3 \mu \mathrm{m}$ in diameter for the deposition of silver, the detection limit could be lowered to $9.2 \mathrm{nM}$. However, $\Delta E$ realized by this method was not large. Therefore, in the second method, one-end of the wire connected to flow channel B was replaced with nickel or zinc. In this case, much larger changes were recorded compared with the case of $\mathrm{Ag} / \mathrm{AgCl}$. Alternatively, a voltage can be applied between the two flow channels through silver wires with $\mathrm{AgCl}$ at the ends using a voltage source. As in the second case, the output charge increased by increasing the voltage applied between the two flow channels. These methods accelerate the conversion of the analyte to silver and will be effective in improving the performance of the device further.

\section{Conclusions}

Coulometry is beneficial for the analysis of components in a solution of a very small volume. Sensitivity and detection limit can be improved by appropriately designing the working electrode structure, coupling the oxidation of an analyte with deposition of silver, and replacing a liquid junction with a metal wire. With these techniques, microfabricated coulometric devices of high performance could be realized.

\section{References}

[1] F. Sassa, H. Laghzali, J. Fukuda, H. Suzuki, Coulometric Detection of Components in Liquid Plugs by Microfabricated Flow Channel and Electrode Structures, Analytical Chemistry 82, 8725-8732 (2010); doi: 10.1021/ac102289a

[2] K. Ikemoto, T. Seki, S. Kimura, Y. Nakaoka, S. Tsuchiya, F. Sassa, M. Yokokawa, H. Suzuki, Microfluidic Separation of Redox Reactions for Coulometry Based on Metallization at the Mixed Potential, Analytical Chemistry 88, 9427-9434 (2016); doi: 10.1021/acs.analchem.6b01234 